\title{
КАИНИЧЕСКИЙ САУЧАЙ CASE REPORT
}

https://doi.org/10.17816/mechnikov201810465-72

\section{АЕТСКАЯ ФЕБРИАЬНО ПРОВОЦИРУЕМАЯ ЭПИАЕПТИЧЕСКАЯ ЭНЦЕФААОПАТИЯ (FIRES-СИНАРОМ)}

\author{
А.М. Щугарева', 2, О.В. Потешкинат, 2, М.К. Кручина', М.А. Русак ${ }^{1}$ \\ ${ }^{1}$ ГБУЗ «Детская городская больница № 1», Санкт-Петербург; \\ ${ }^{2}$ ФГБОУ ВО «Северо-Западный государственный медицинский университет им. И.И. Мечникова» \\ Минздрава России
}

Аля цитирования: Шугарева А.М., Потешкина О.В., Кручина М.К., Русак М.А. Аетская фебрильно провоцируемая эпилептическая энцефралопатия (FIRES-синдром) // Вестник Северо-Западного государственного медицинского университета им. И.И. Мечникова. - 2018. - Т. 10. - № 4. - С. 65-72. https://doi.org/10.17816/mechnikov201810465-72 Поступила: 02.10.2018

ОАобрена: 16.11.2018

Принята: 07.12.2018

- Лихорадочно провоцируемый синдром злокачественной эпилептической энцефалопатии у детей (Fever infection-related epilepticy syndrome - FIRES) - редкое, тяжелое постинфекционное/постлихорадочное неврологическое расстройство, которое представляет собой рефрактерный к терапии эпилептический статус у ранее здорового ребенка. Этиология FIRES остается неизвестной, предполагаются поствоспалительные и аутоиммунные механизмы развития заболевания. Выздоровление возможно, но у большинства детей развивается выраженное нарушение когнитивно-познавательной деятельности и резистентная к терапии эпилепсия.

- Ключевые слова: эпилептическая злокачественная энцефалопатия; лихорадка; дети.

\section{FEVER-INDUCED EPILEPTIC ENCEPHALOPATHY IN CHILDREN (FIRES)}

\author{
L.M. Schugareva', 2 , O.V. Poteshkina'1,2, M.K. Kruchina ${ }^{1}$, M.A. Rusak ${ }^{1}$ \\ ${ }^{1}$ Children's Hospital No. 1, St. Petersburg, Russia; \\ ${ }^{2}$ North-Western State Medical University named after I.I. Mechnikov, Saint Petersburg, Russia
}

For citation: Schugareva LM, Poteshkina OV, Kruchina MK, Rusak MA. Fever-induced epileptic encephalopathy in children (FIRES). Clinical case. Herald of North-Western State Medical University named after I.I. Mechnikov. 2018;10(4):65-72. https://doi.org/10.17816/mechnikov201810465-72

Received: October 2, 2018

Revised: November 16, 2018

Accepted: December 7, 2018

- Fever infection-related epilepticy syndrome, FIRES - rare severe epileptic encephalopathy infection or fever-induced refractory epileptic status developing in a previously healthy child. The etiology of FIRES remains unknown; post-inflammatory and autoimmune mechanisms are assumed. Recovery is possible, but the majority of children develop significant cognitive failure and refractory epilepsy.

- Keywords: malignant epileptic encephalopathy; fever; children.

FIRES (Febrile infection-related epilepsy syndrome) - синдром тяжелой эпилептической энцефалопатии у детей школьного возраста, индуцированный предшествующей инфекцией или лихорадкой. По данным литературы, FIRES встречается с частотой 1-9/100 000 детского населения [1-3].
Первое описание клинического случая FIRES опубликовано Lyon et al. (1961) [4]. Интерес к проблеме постнатального снижения когнитивных функций до сих пор остается актуальным [5-9]. В большинстве случаев авторы предполагают основной причиной заболевания инфекционное поражение паренхимы мозга на 
фоне фебрильной лихорадки [8-11]. Для обозначения данного вида неврологических расстройств в литературе используются различные термины: «острая энцефалопатия неясного происхождения у младенцев и детей» (acute encephalopathy of obscure origin in infants and children); «острый энцефалит с рефрактерными, повторными парциальными приступами» (acute encephalitis with refractory, repetitive partial seizures - AERRPS); «острая энцефалопатия с поствоспалительным индуцированным разрушительным эпилептическим статусом у детей школьного возраста» (acute encephalopathy with inflammation-mediated status epilepticus devastating epilepsy in school-aged children - DESC); «идиопатическая катастрофическая эпилептическая энцефалопатия» (idiopathic catastrophic epileptic encephalopathy); «идиопатическая гемиконвульсивная гемиплегия и эпилептический синдром» (idiopathic hemiconvulsion-hemiplegia and epilepsy syndrome - IHHE); «лихорадочно индуцированная эпилептическая энцефалопатия у детей школьного возраста» (fever-induced refractory epileptic encephalopathy in school-age children); «впервые в жизни развившийся рефрактерный эпилептический статус (new-onset refractory status epilepticus - NORSE) и др. $[3,6-9,11-13]$. При всех синдромах в постлихорадочном периоде развивались рефрактерные к стандартной противоэпилептической терапии судороги с неблагоприятным неврологическим исходом $[3,14,15]$.

Возраст дебюта FIRES варьирует от 2 до 17 лет, развиваясь преимущественно у детей школьного возраста (медиана -8 лет). Среди заболевших незначительно преобладают мальчики. Синдром проявляется эпилептическими приступами (ЭП) у ранее здоровых детей при наличии лихорадки или спустя несколько дней после ее начала. В качестве триггера называют инфекцию верхних дыхательных путей (59 \%), диспепсический эпизод (7\%), в $39-40 \%$ причина остается неизвестной. Продолжительность лихорадки с момента начала заболевания до развития судорог составляет 2-14 дней (в среднем - 5 дней) [16]. Для FIERS характерен клинический полиморфизм и бифазное течение [3, 17]. ЭП имеют фокальный моторный двусторонний или генерализованный тонико-клонический характер и в течение 24-36 часов трансформируются в эпилептический статус (ЭС). В ряде случае описывают миоклонические ЭП с вовлечением мышц лица, конечностей, передней брюшной стенки [15]. ляющую, по различным источникам, 2-40 дней (в среднем - 16 дней) [3, 15-17].

Для острой фазы заболевания характерны выраженные автономные расстройства: бледность, апноэ, цианоз, злокачественная гипертермия, артериальная гипо/гипертензия $[15,18]$.

Патогенез FIRES неизвестен. В большинстве случаев заболевание имеет бифазное течение, что подтверждает воспалительную этиологию [16]. В качестве основного патогенетического фактора рассматривается двустороннее поражение гиппокампов, формирующееся в результате продолжительного эпилептического статуса и системного воспалительного ответа $[3,17,19]$. В результате в крови увеличивается количество глутамина, цитокинов, простагландинов и лейкотриенов, что приводит к изменению проницаемости ионных каналов и формированию стойких эпилептических очагов в головном мозге [13]. Спектр изучаемых инфекционных агентов, являющихся возможными триггерами этого процесса, включает вирусы простого герпеса, герпеса человека 6-го типа, аденовирусы, парвовирус В19, энтеровирусы, риновирусы, вирусы гриппа и парагриппа, кори, эпидемического паротита, краснухи, клещевые вирусы, ретровирусы [3]. Вместе с тем инфекционная этиология FIERS не подтверждена. Высказывается предположение о развитии воспалительно-опосредованной каналопатии, которая приводит к формированию устойчивых эпилептических очагов $[2,3]$. При этом ЭС при FIRES рефрактерен к иммунотерапии, включая высокие дозы стероидов, внутривенному иммуноглобулину и плазмаферезу. Поиск вероятных метаболических расстройств заболевания продолжается. Описаны случаи поломки в гене $\mathrm{PCDH} 19$ на $\mathrm{X}$-хромосоме, что является патогномоничным для нарушения функции GLUT-транспортера и отчасти объясняет положительное влияние на течение заболевания кетогенной диеты (КД) $[13,18]$.

В исследовании R.H. Caraballo et al. у пациентов c FIERS осуществляли поиск аутоиммунных нарушений: определяли титр антител к калиевым каналам (VRRL-Ab), глютаматдекарбоксилазе (анти-GAD-антитела), рецепторам глутамата (анти-GLUR3-антитела) и олигоклональным антителам в цереброспинальной жидкости (ЦСЖ) [15]. Получены положительные результаты в 25-40 \% случаев, что не является патогномоничным для FIERS [19].

Плеоцитоз в ЦСЖ может быть выявлен в дебюте заболевания, что имитирует развитие энцефалита. Плеоцитоз в ЦСЖ составляет 
2-42 клетки (в среднем - 5 клеток), количество белка и глюкозы в норме. Вместе с тем инфекционного агента не находят как в жидких средах организма, так и при биопсии мозга.

При проведении МРТ в остром периоде характерных воспалительных изменений в веществе мозга не выявляют (41 \%) [3]. В 41 \% случае регистрируют односторонние или двусторонние изменения сигнала на Т2 ВИ от гиппокампов, периинсулярных, лобно-париетальных областей, а также диффузные изменения по типу отека-набухания коры или таламусов. В единичных случаях диагностировали паренхиматозное кровоизлияние в мозжечок [20]. В хронической стадии FIERS у 50-65 \% пациентов формируются атрофия и астроглиоз (мезиальный темпоральный склероз) как исход затяжной эпилептической энцефалопатии, что коррелирует с тяжестью и продолжительностью ЭС (рис. 1).

В $20 \%$ МРТ головного мозга остается либо нормальной, либо регистрируют парадоксальную нормализацию МР-изображения по окончании острого периода [3].

На ЭЭГ выявляют паттерн «вспышка - подавление» (41\%), фокальную эпилептиформную активность (59-75\%) или диффузное замедление биоэлектрической активности (41 \%). Характерно формирование эпилептического электрического ЭС во время сна $(47 \%)[2,3]$. В дальнейшем индекс эпилептической активности уменьшается до $30 \%$.

\section{Аечение и прогноз}

Выживаемость после FIERS составляет 85-90 \%, летальность достигает $9 \%$. После завершения острой стадии заболевания и восстановления сознания дети практически заново учатся ходить, говорить и мыслить. Этот процесс может продолжаться от нескольких недель до нескольких месяцев. Полное выздоровление фиксируют у 10 \% детей. Во всех случаях длительно сохраняются расстройства автономной нервной системы. В $90 \%$ развивается фармакорезистентная эпилепсия, без «светлого» промежутка после ЭС. Оценка когнитивных функций в хронической стадии FIERS: нормальные когнитивные функции определяются у 20 \% выживших пациентов, пограничные показатели у $16 \%$, легкая умственная отсталость - у $14 \%$, умеренная умственная отсталость - у $24 \%$, тяжелая степень - у $12 \%$, вегетативный статус у $16 \%$. Длительность хронической стадии FIERS в среднем составляет 5 лет $[2,3]$. В легких случаях у детей развивается специфический ней-

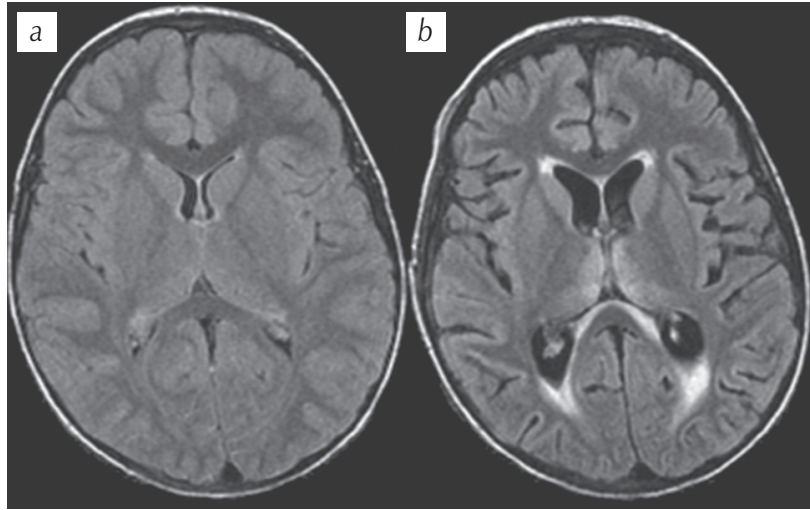

Рис. 1. Пациент, 4 года. Диагноз: FIRES: $a$ - аксиальный срез, МРТ, Т1 ВИ на 1-й день госпитализации без патологии; $b-$ аксиальный срез, МРТ, Т1 ВИ на 30-й день госпитализации - нарастание атрофии, повышение сигнала от таламусов и перивентрикулярных отделов наружных отделов боковых желудочков (J Perumpillichira. 2014)

Fig. 1. Patient, 4 y. o. Diagnosis: FIRES: $a-$ axial fluid attenuated inversion recovery image on day 1 of hospitalization shows no abnormalities; $b$ - axial fluid attenuated inversion recovery image on day 30 of hospitalization widening of sulci and lateral ventricles suggestive of diffuse cerebral atrophy, hyperintense signal changes are seen in bilateral thalami as well as along the ependymal margins of the ventricles and peri-atrial regions (J Perumpillichira. 2014)

ропсихологический синдром, обусловленный поражением височных долей и проявляющийся снижением кратковременной слуховой, зрительной памяти, гиперактивным поведением. Типично развитие лобного дизэкзекутивного синдрома - нарушение мотивационной сферы, речевой регуляции произвольной деятельности, выработки планов и построения программ поведения. Отмечено, что в случае эпилепсии с паттерном лобного дизэкзекутивного синдрома значительно нарушается социальная адаптация и способность к обучению [11].

Специфическое лечение не разработано. Терапия включает посиндромное трехэтапное лечение: барбитуровая кома, ИВЛ, высокие дозы стероидов, в/в иммуноглобулины, плазмаферез. Из препаратов применяют барбитураты, лидокаин, пиридоксин, биотин, магний, верапамил, филиновую кислоту, в особо затяжных случаях - ритуксимаб. Доказательства эффективности использованных схем терапии основаны на описанных клинических случаях и имеют эмпирический, несистематизированный характер из-за низкой частоты встречаемости данной патологии [17]. В ряде исследо- 
ваний описана эффективность КД. Изучали различные механизмы действия КД, включая воздействие на нейротрансмиттеры или их рецепторы, а также противовоспалительные или антиэксайтотоксические эффекты $[17,19]$. Однако нет научно обоснованных рекомендаций по использованию КД при FIERS. Абсолютные противопоказания к КД включают такие метаболические расстройства, как дефицит пируваткарбоксилазы, дефекты окисления жирных кислот, включая транспортер карнитина, дефекты и первичный дефицит синтеза карнитина [18]. Гиперлипидемия и нефролитиаз служат относительными противопоказаниями для проведения КД [21]. В хронической стадии прибегают к нейрохирургическому лечению, аналогичному при фармакорезистентных формах эпилепсии [22].

\section{Клинический случай}

Девочка С.К., 15 лет, поступила экстренно в связи со статусным течением судорог. Из анамнеза известно: росла и развивалась по возрасту. Ранее судорог не было. Наследственность по эпилепсии не отягощена. За 10 дней, во время загородной поездки, перенесла острое лихорадочное заболевание с абдоминальным синдромом, получала симптоматическое лечение.

Судороги развились 08.05.18 на фоне полного здоровья, генерализованные тонико-клонические, серийное течение, длительностью 40 минут. Купированы внутривенным введением реланиума на догоспитальном этапе. При поступлении: состояние тяжелое, медикаментозно седатирована. Шкала комы Глазго (ШКГ) 9 баллов. Спонтанный горизонтальный нистагм. Зрачки $D=S$, ФТР (+). Мышечный тонус диффузно снижен. Сухожильные рефлексы снижены, $D=S$. Патологических стопных знаков нет. Менингеальных симптомов нет. Выполнена МРТ головного мозга - патологии не выявлено. 09.05.18 (1-е сутки) - сохраняются вялость, сонливость. Пробуждается после болевого и тактильного раздражителя (ШКГ 10 баллов), простые команды выполняет. В контакт вступает избирательно. На вопросы отвечает односложно, неохотно, быстро истощается. Артикуляция «смазанная». 10.05 .18 (2-е сутки) - рецидив тонико-клонических судорог c утратой сознания. Повторно введен реланиум. В терапию введен леветирацетам 1000 мг/сут. ЭЭГ (10.05.18) (2-е сутки) - диффузная дезорганизация фонового ритма бета-активностью ная лихорадка, гектический тип, тахикардия, артериальная гипертензия. Прогрессирование ЭС - серийное течение по типу миоклоний в конечностях, мышцах передней брюшной стенки, с переходом в генерализованные тонико-клонические. Введение реланиума - неэффективно, сознание утрачено. Пациентка введена в состояние барбитуровой комы с помощью тиопентала натрия в дозе 4,6 мг/кг в час, переведена на ИВЛ. Дифференциальный диагноз проводился с вирусным энцефалитом, дебютом острого демиелинизирующего энцефаломиелита, аутоиммунным лимбическим энцефалитом с антителами к NMDA-рецепторам, метаболической энцефалопатией. Выполнена диагностическая люмбальная пункция. ЦСЖ 11.05 .18 (3-и сутки) - цитоз 2/3, белок 0,46 г/л, глюкоза 3,1 (все показатели в норме); ПЦР к вирусам семейства герпеса - результат отрицательный, посев - нет роста. NMDA-антитела - не выявлены. На фоне барбитуровой комы судороги купированы, но при попытке снизить дозу до 3 мг/кг в час - новый рецидив ЭС.

ЭЭГ (15.05.18) (7-и сутки) - выраженная отрицательная динамика, нарастание диффузных изменений (замедление, дезорганизация фонового ритма, появление эпилептиформной активности в правой лобно-височной области в виде комплексов «острая волна - медленная волна» (рис. 2, b). В связи с отрицательной динамикой по данным ЭЭГ и отсутствием улучшения состояния выполнена повторная МРТ головного мозга с контрастированием - патологии не выявлено. 16.05 .18 (8-е сутки) выполнена повторная люмбальная пункция. ЦСЖ - цитоз 1/3, белок 0,14 г/л (все показатели в норме). Посев - нет роста. Проводили антибактериальную терапию (роцефин), метаболическую терапию (цитофлавин).

На 8-е сутки заболевания - отмена тиопентала натрия. Состояние остается тяжелым, сохраняется фебрильная лихорадка $38,4^{\circ} \mathrm{C}$, тахикардия до 109 в минуту, гипергидроз, АД 120/65 мм рт. ст. На болевой раздражитель вегетативная реакция (учащенное дыхание, гиперемия кожных покровов). Зрачки $D=S$, ФТР (+). Расходящееся косоглазие. Диффузная мышечная гипотония. Сухожильные рефлексы снижены, $D=S$. Брюшные (-). Положительный симптом Бабинского с двух сторон. Появились рефлексы орального автоматизма.

С 17.05.18 (9-е сутки заболевания) состояние улучшилось, экстубирована. Судороги больше не повторялись. Девочка продолжает фебрильно лихорадить, максимально до $38,6^{\circ} \mathrm{C}$. 

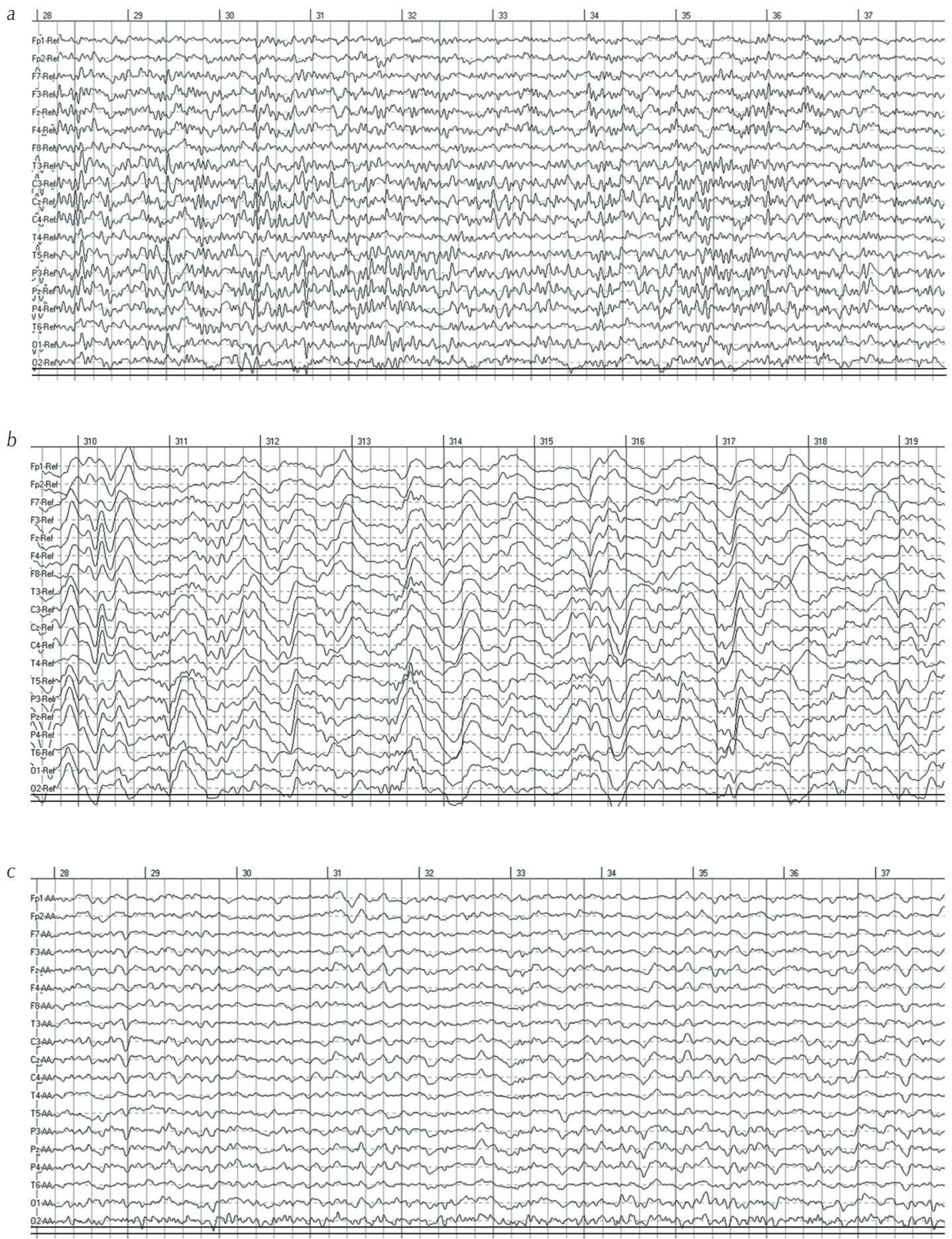

Рис. 2. Пациентка С.К., 15 лет. Диагноз: FIRES: $a$ - ЭЭГ в 1-е сутки заболевания - диффузные изменения в виде дезорганизации фонового ритма избыточной бета-активностью; $b-$ ЭЭГ на 5-е сутки заболевания - диффузное замедление фонового ритма, в правой лобно-височной области фиксируются комплексы «острая волна - медленная волна»; $c$ - ЭЭГ перед выпиской (20-е сутки заболевания) - отчетливая положительная динамика, умеренное замедление фонового ритма, исчезновение эпилептиформной активности на фоне лечения. Собственное наблюдение

Fig. 2. Patient S.K., 15 y. o. Diagnosis: FIRES: $a$ - EEG (1 day of the disease) - diffuse disorganization of rhythm beta activity; $b$ - EEG (day 5 of the disease) - a diffuse slowing of the background activity, in the right frontal-temporal region sharp-and-slow wave epileptiform activity are registered; $c$ - EEG before discharge (day 20 of the disease) - the improvement of the background activity, moderate slowing of the background activity, no epileptiform activity recorded. Own observation 




Рис. 3. Температурный лист. Гектический тип лихорадки Fig. 3. Temperature sheet. Hectic type of fever

Периодически беспокойна. Сохраняется повышенная потливость, эпизоды тахикардии до 110 в минуту. АД 90/60 мм рт. ст. Сознание восстановилось до уровня ШКГ 9 баллов: начала вступать в контакт, выборочно отвечать на вопросы, но быстро истощается, выраженная вялость, заторможенность, «смазанность» речи.

18.05.18 (10-е сутки заболевания) переведена в отделение. При переводе: состояние средней тяжести. Лихорадит субфебрильно. Отмечается повышенная истощаемость (физическая, психическая). Не может выстроить программу действий (бытовые функции), требуется постоянный контроль взрослого. Ретроградная амнезия. Речь внятная, замедленная, односложная. Ходит неуверенно, несколько шагов, с поддержкой, в пределах палаты. Мышечная сила в конечностях -4 балла, грубых парезов нет. Сухожильные рефлексы высокие, с ног с элементами клонусов, $D=S$. Координационная неловкость, туловищная атаксия. Патологических симптомов нет. Выраженная потливость, артериальная гипотензия. Тахикардия ортостатического типа до 110 при вертикализации. Нарушений трофики нет. Физиологические отправления в норме.

В отделении проведены следующие обследования:

- клинический анализ крови, биохимический анализ крови - без патологии;

- ПЦР (кровь, ЦСЖ) на вирусную панель отрицательно;

- ИФА к вирусу Эпштейна - Барр (ВЭБ) капсидный антиген 0,605; ранние белки - (антитела к хронической ВЭБ-инфекции) от 08.05.18;

- кислотно-основное состояние - компенсировано; лактат сыворотки крови 2,2-1,6 ммоль/л (норма - до 2,0 ммоль/л);

- ТТГ - 3,29; Т4 - 85; Т3 - 1,51 (все показатели в норме);

- УЗИ органов малого таза, брюшной полости, почек - без патологии;

- ЭЭГ 25.05.18 (17-е сутки заболевания) умеренное замедление фонового ритма, без очаговых изменений и эпилептиформной активности (рис. 2, c);

- ЭКГ - миграция водителя ритма по предсердиям. ЧСС - 63 в минуту.

В отделении пациентка получала лечение: инфузионную терапию, базисную антиэпилептическую терапию (леветирацетам, депакин), преднизолон в/в капельно № 5, ибупрофен, симптоматическое лечение.

На фоне терапии состояние улучшилось. Длительно сохранялась лихорадка, которую купировали стероидной терапией с 17-х суток заболевания (рис. 3). К выписке сохраняется выраженная астенизация, вегетативные расстройства. В отделении отменена антиконвульсантная терапия - в раннем восстановительном периоде судороги не рецидивировали. Выписана на 20-е сутки заболевания.

\section{Обсуждение}

Для постановки диагноза FIERS применяют основные характеристики:

1) развитие заболевания у ранее здоровых детей в возрасте от 3 до 17 лет;

2) острое начало в виде лихорадки и симптомов инфекционного эндотоксикоза;

3) развитие фокальных эпилептических приступов с серийным течением, чаще через несколько дней/недель после острого лихорадочного периода;

4) отсутствие инфекционного возбудителя при исследовании ЦСЖ, крови или других жидкостей и тканей организма;

5) в хронической фазе в $90 \%$ случаев развивается фармакорезистентная эпилепсия;

6) у большинства больных после окончания острого периода развиваются стойкие неврологические нарушения (поведения, памяти, внимания). В 10 \% случаев пациенты возвращаются к исходному уровню.

Диагноз был поставлен на основании соответствия течения болезни следующим критериям FIRES: возраст пациентки 15 лет; наличие 
эпизода лихорадки за 10 дней до дебюта судорог; начало с судорог с переходом в длительный эпилептический статус (9 суток); отсутствие возбудителя инфекционного процесса в сыворотке крови и ЦСЖ. По причине неэффективности стандартной противоэпилептической терапии инициировано введение пациентки в тиопенталовый наркоз, выход из которого сопровождался нарушением двигательных навыков и развитием лобного дизэкзекутивного синдрома - нарушением мотивационной сферы, речевой регуляции произвольной деятельности, выработки планов и построения программ поведения. Особенностью данного случая является хороший ранний неврологический исход с развитием легких когнитивных и двигательных нарушений. Вместе с тем формирование стойкого неврологического исхода заболевания, в том числе эпилепсии, может происходить в более поздние сроки. Это делает необходимым динамическое наблюдение за больными с участием эпилептолога и психоневролога в течение последующих 5 лет.

\section{Список митературы}

1. Бобылова М.Ю. Резистентные фрормы эпилепсии, ассоциированные с фебрильно провоцируемыми приступами (синдромы Араве, DESCV, HНE) (^екция) // Русский журнал Аетской неврологии. - 2012. - T. VII. № 4. - C. 31-40. [Bobylova MY. Resistant epilepsy forms, associated with fever-provoked (febrile) epileptic seizures (Dravet syndrome, DESCV, HHE) (lecture). Russian Journal of Child Neurology. 2012;7(4):31-40. (In Russ.)]. https:// doi.org/10.17650/2073-8803-2012-7-4-31-40.

2. Шалькевич А.В., Аьвова О.А., Кудлач А.И., Комир В.В. Особенности и характер течения у детей синдрома эпилепсии, индуцированного фебрильной инфекцией (FIRES) // Неврология, нейропсихиатрия, психосоматика. - 2014. - Т. 6. - № 4. - C. 65-71. [Shalkevich LV, Lvova OA, Kudlach Al, Komir VV. The specific features and pattern of febrile infection-related epilepsy syndrome (FIRES) in children. Neurology, Neuropsychiatry, Psychosomatics. 2014;6(4):65-71. (In Russ.)]. https://doi. org/10.14412/2074-2711-2014-4-65-71.

3. Andreas Van Baalen, Martin Häusler, Rainer Boor, et al. Febrile infection-related epilepsy syndrome (FIRES): a nonencephalitic encephalopathy in childhood. Epilepsia. 2010;51(7):1323-1328. https://doi.org/10.1111/j.15281167.2010.02535.x.

4. Lyon G, Dodge PR, Adams RD. The acute encephalopathies of obscure origin in infants and children. Brain. 1961;84(4):680708. https://doi.org/10.1093/brain/84.4.680.

5. Hington GG. Postnatal organic causes of mental retardation. Canad Med Ass J. 1962;87(9):501-507.
6. Baxter P, Clarke A, Cross $\mathrm{H}$, et al. Idiopathic catastrophic epileptic encephalopathy presenting with acute onset intractable status. Seizure. 2003;12(6):379-87. https://doi. org/10.1016/S1059-1311(02)00340-0.

7. Mikaeloff Y, Jambaque I, Hertz-Pannier L, et al. Devastating epileptic encephalopathy in school-aged children (DESC): a pseudo encephalitis. Epilepsy Res. 2006;69:67-69. https://doi.org/10.1016/j.eplep- syres.2006.01.002.

8. Sakuma H, Awaya Y, Shiomi M, et al. Acute encephalitis with refractory, repetitive partial seizures (AERRPS): a peculiar form of childhood encephalitis. Acta Neurol Scand. 2010;121(4):251-6. https://doi.org/10.1111/ j.1600-0404.2009.01198.x.

9. Sakuma H, Fukumizu M, Kohyama J. Efficacy of anticonvulsants on acute encephalitis with refractory, repetitive partial seizures (AERRPS). No To Hattatsu. 2001;33(5):385-90.

10. Kramer U, Shorer Z, Ben-Zeev B, et al. Severe refractory status epilepticus owing to presumedencephalitis. J Child Neurol. 2005;20(3):184-7. https://doi.org/10.1177/08830 738050200030301.

11. Мухин К.Ю., Петрухин А.С., Холин А.А. Эпилептические энцефралопатии и схожие синдромы у детей. М.: АртСервис ^тА, 2011. - 680 с. [Mukhin KY, Petrukhin AS, Kholin AA. Epileptic encephalopathies and similar syndromes at children. Moscow: ArtServis Ltd; 2011. 680 p. (In Russ.)]

12. Wilder-Smith E, Lim E, Teoh $\mathrm{H}$, et al. The NORSE (New-onset Refractory Status Epilepticus) Syndrome: Defining a Disease Entity. Ann Acad Med Singapore. 2005;34:417-20.

13. Nabbout R. FIRES and IHHE: Delineation of the syndromes. Epilepsia. 2013;54(6):54-6. https://doi.org/10.1111/ epi.12278.

14. Sahin M, Menache CC, Holmes GL, Riviello JJ. Outcome of severe refractory status epilepticus in children. Epilepsia. 2001;42(11):1461-7. https://doi.org/10.1046/j.15281157.2001.21301.x.

15. Caraballo RH, Reyes G, Lopez Avaria MF, et al. Febrile infection-related epilepsy syndrome: a study of 12 patients. Seizure. 2013;22(7):553-9. https://doi.org/10.1016/j.seizure.2013.04.005.

16. Maegaki Y, Kondo A, Okamoto R, et al. Clinical characteristics of acute encephalopathy of obscure origin: a biphasic clinical course is a common feature. Neuropediatrics. 2006;37:269-277. https://doi.org/10.1055/s-2006-955928.

17. Kramer U, Chi CS, Lin KL, et al. Febrile infection-related epilepsy syndrome (FIRES): pathogenesis, treatment, and outcome: a multicenter study on 77 children. Epilepsia. 2011;52(11):1956-65. https://doi.org/10.1111/j.15281167.2011.03250.x.

18. Евтушенко С.К. Разрушительные и труднокурабельные формы эпилепсии и эпилептические энцефралопатии у Аетей // Международный неврологический журнал. - 2012. - № 6 (52). - C. 15-26. [Evtushenko SK. Destructive and difficult-to-treat epileptic forms and epi- 
leptic encephalopathies in children. Mezhdunarodnyi nevrologicheskii zhurnal. 2012;6(52):15-26. (In Russ.)]

19. Specchio N, Fusco L, Claps D, Vigevano F. Epileptic encephalopathy in children possibly related to immune-mediated pathogenesis. Brain Dev. 2010;32(1):51-56. https:// doi.org/10.1016/j.braindev.2009.09.017.

20. Perumpillichira Cherian, Katherine Nickels. Febrile infection-related epilepsy syndromes and their treatment. Journal of Pediatric Epilepsy. 2014;3:67-74. https://doi. org/10.3233/PEP-14074.

- Адрес автора для переписки (Information about the author)

Тел. / Tel.: +7(921)3352637

E-mail: ovpoteshkina@gmail.com

SPIN-код: 9407-5169

21. Харитонов В.И. Новое в эпилептологии // Нейро News: психоневрология и нейропсихиатрия. - 2013. № 7(52). - C. 10-11. [Kharitonov VI. New in an epileptologiya. Neiro News: psikhonevrologiya i neiropsikhiatriya. 2013;7(52):10-11. (In Russ.)]

22. Kim DW, Kim KK, Chu K, Lee SK. Surgical treatment of delayed epilepsy in hemiconvulsion-hemiplegiaepilepsy syndrome. Neurology. 2008;70(22 Pt 2):2116-22. https:// doi.org/10.1212/01.wnl.0000289192.50924.93. 